L. E. Visser - A. E. Vlug · J. van der Lei

B. H. Ch. Stricker

\title{
Cough due to ace inhibitors: a case-control study using automated general practice data
}

Received: 1 March 1995/Accepted in revised form: 4 May 1995

\begin{abstract}
Objectives: To determine the risk of coughing as an adverse reaction to ACE inhibitors under everyday circumstances in a large population, and to study whether this adverse effect was duration or dose dependent.

Design: A population-based case-control study.

Setting: Ten general practices of 14 Dutch general practitioners (GP), in which all consultations, morbidity and medical interventions, including drugs prescribed, were registered over the 18 month period from 1st September, 1992 to 1st March, 1994.

Subjects: 1458 patients with incident coughing and up to four controls per case were obtained (total 4182 controls), matched for GP. All cases and controls were 20 years or older and had no record of respiratory infection, influenza, tuberculosis, asthma, chronic bronchitis, emphysema, congestive heart failure, sinusitis, laryngitis, haemoptysis or respiratory neoplasms during the study period.

Results: Cases were 2.1-times more likely than controls to have been exposed to ACE inhibitors $(95 \% \mathrm{CI}$ $1.5-3.1)$, but after adjustment the odds ratio was 1.4 (95\% CI 0.9-2.1). The crude odds ratio for captopril was $1.3(95 \%$ CI $0.7-2.5)$, for enalapril $2.6(95 \% \mathrm{CI}$ 1.6-4.2) and for lisinopril $2.0(95 \%$ CI $0.5-9.3)$. The adjusted odds ratio for captopril was $0.9(95 \% \mathrm{CI}$ 0.4-1.7), for enalapril 1.7 (95\% CI 1.03-2.8) and for lisinopril 1.7 (95\% CI 0.4-7.9). For patients who had been on ACE inhibitor treatment for no longer than 2
\end{abstract}

\section{E. Visser · B. H. Ch. Stricker $(\triangle \triangleleft)$}

Department of Epidemiology and Biostatistics,

Erasmus University Medical School, room Ee 2126

Dr. Molewaterplein 50, NL-3015 GE Rotterdam,

The Netherlands

B. H. Ch. Stricker

Drug Safety Unit, Inspectorate for Health Care, Rijswijk,

The Netherlands

A. E. Vlug $\cdot$ J. van der Lei

Department of Medical Informatics, Erasmus University,

Rotterdam, The Netherlands months the odds ratio was $4.8(95 \%$ CI 1.7-13.3). The odds ratio declined to 2.0 (95\% CI 1.1-3.8) for those who had taken an ACE inhibitor for 2-6 months, and to $1.6(95 \% \mathrm{CI} 0.9-2.7)$ for those on ACE-inhibitors for more than 6 months.

Conclusion: The risk of coughing was increased twofold among ACE inhibitor users, but the odds ratios were no longer significant after controlling for several confounding factors. The risk of developing cough due to ACE-inhibitors declines with the duration of treatment, possibly due to depletion of susceptible persons.

Key words ACE inhibitors; cough, epidemiological study, general practice

\section{Introduction}

Coughing due to angiotensin converting enzyme (ACE) inhibitors was first reported more than 10 years ago in association with captopril $[1,2]$. Other ACE-inhibitors were subsequently shown to increase the risk of coughing. Several mechanisms have been postulated for ACE inhibitor-induced cough, including for instance, increased bronchial reactivity, enhanced cough reflex, prostaglandin-, and bradykinin-mediated effects and stimulation of pulmonary C-fibre receptors by elevated levels of substance P [3]. Several risk factors have been suggested, for instance, dose, duration of treatment, age and type of ACE-inhibitor, and coughing seems to be relatively more frequent in women [4-21]. The latter is in line with an earlier case-control study by the authors, in which the risk of coughing was higher in females than in males [22].

The reported frequency of cough due to $A C E$ inhibitors has varied widely depending on the population under study and the methodological approach used. Two large-scale postmarketing studies of unselected patient populations yielded much lower estimates of the incidence than studies in hospital or tertiary clinics $[23,24]$. In some studies, the frequency of cough to 
ACE inhibitors has been expressed as an incidence rate, whereas in others cumulative incidences and prevalence estimates have been used. It has been suggested that the risk of coughing may be higher with ACE-inhibitors with a long duration of action [3]. In an earlier study with another data base, we found a higher risk of coughing due to enalapril than to captopril [22]. As the study period was limited to 3 months, however, we were not able to examine any duration-effect relationship.

The main aims of the current study were to obtain a risk estimate of coughing due to ACE inhibitors under everyday circumstances, in a large population over a longer period, and to examine whether the effect was dependent on dosage and duration of therapy.

\section{Subjects and methods}

\section{Data source}

Data for the study came from the project for Integrated Primary Care Information (IPCI), a collaboration between the Department of Medical Informatics of the Erasmus University Medical School Rotterdam (MIEUR) and the Institute for Medical Statistics in the Hague. One of the purposes of IPCI is to conduct postmarketing surveillance studies using data from computer-based patient records of general practitioners (GPs). In 1992, 58\% of Dutch GPs had installed an information system and $38 \%$ had purchased and implemented a patient-record module [25]. Initially, only users of the ELIAS information system have participated in the project, but other systems will be included later. At MIEUR, the data are entered into a relational database management system.

The events recorded include administrative data about all registered patients and summaries of every visit of a patient to a GP: details of patient complaints, laboratory and other tests and their results, diagnoses, referrals to other health care providers and medical interventions, including prescribed drugs. For coding the reason for the encounter and the diagnoses, ELIAS supports the International Classification of Primary Care (ICPC) [26]. Of the recorded diagnoses, $73 \%$ had a diagnostic ICPC code, and the remainder were registered in free text. A database of all drugs available in the Netherlands, maintained by the Royal Dutch Association for the Advancement of Pharmacy, enables the coding of prescriptions, according to the Anatomical Therapeutic Chemical (ATC) classification scheme recommended by the World Health Organisation [27]. The data used for the study were collected from 10 general practices in the period from 1st September, 1992 to 1st March, 1994. These 10 practices were considered to be representative of GP standards, prescription profile and morbidity.

\section{Design and case definition}

In order to determine the risk of coughing as an adverse reaction to ACE inhibitors, we conducted a case-control study. Patients aged 20 years and older, who consulted a GP for cough (ICPC code R05, or diagnosis 'cough' recorded in free text) for the first time during the study period (incident cases), were potentially eligible as case patients. For case patients who had two or more incident episodes of coughing during the study period, only the first episode was considered. Four controls per case were randomly selected from patients who had had at least one consultation with the same GP office as the case during the study period, and who had not reported coughing during that period. Cases and controls were excluded if any of the following diseases were registered: respiratory infections, influenza tuberculosis, asthma, chronic bronchitis, emphysema, congestive heart failure, sinusitis, laryngitis, haemoptysis, respiratory neoplasms, or other conditions known to cause or predispose to coughing.

\section{Data analysis}

The date of the first consultation for coughing of the case was taken as the index date and was assigned to each control to ensure that the period of possible exposure to the drug of interest was identi$\mathrm{cal}$ in cases and controls, and to control for seasonal variation in morbidity and prescriptions. Cases and matched controls had exactly the same length of medical history prior to the index date. All control subjects had to have had at least one consultation after the index date of the case to ensure that they were alive and were still present in the catchment area.

For each prescription of an ACE inhibitor a treatment course was calculated from the number of prescribed tablets divided by the prescribed number of tablets per day. If a subject was no longer on ACE inhibitor treatment, the length of the last treatment course was multiplied by the factor 1.1 with a minimum of 2 days and a maximum of 14 days in order to control for carry-over of drug effects. The total exposure experience of each subject was calculated by summarising the duration of all treatment courses. Subjects were defined as users if the index date fell between the start and stop data of the particular ACE-inhibitor.

The odds ratio of exposure to ACE inhibitors amongst patients with cough compared to control patients was calculated as the crude matched odds ratio and the $95 \%$ confidence interval ( $95 \% \mathrm{CI}$ ). For each potential risk factor a crude matched exposure odds ratio was calculated. The following variables were considered as potential confounders: age, gender, number of prescriptions, number of consultations, smoking status and use of NSAID, $\beta$-blockers [28], thiazides [29] or calcium-antagonists [30]. Treatment courses of these drugs were calculated as described above for ACE-inhibitors. Factors that caused a change in the crude odds ratio of use of ACE inhibitors of at least $5 \%$ were included in the multivariate model, applying conditional logistic regression [31] with the EGRET statistical package [32]. Subanalyses were performed for users of captopril, enalapril, lisinopril and perindopril, as well as for females and males, by applying the same multivariate models. We also investigated whether this side effect was dose-dependent, using the same conditional model with 3 dose levels. For captopril and enalapril, but not as yet for other ACE inhibitors, there were sufficient data to evaluate risk according to dose. The effect of duration of exposure was also studied using the same multivariate model.

\section{Results}

In total, 44787 patients were registered in the 10 general practices. Of them, a total of 33779 patients had 291145 consultations with their GP. During the 18 months of the study, the GPs wrote a total of 253880 prescriptions. There was a total of 652 patients in the database who had been prescribed an ACE inhibitor or a combination with an $\mathrm{ACE}$ inhibitor on one or more occasions. 242 had been prescribed captopril or a combination with captopril $(37 \%), 394$ enalapril or a combination $(60 \%), 29$ lisinopril or a combination $(4 \%)$ and 31 perindopril (5\%). Of the 652 patients given prescription for an ACE inhibitor, 181 reported coughing during the study period $(27.8 \%, 95 \%$ CI $24.4 \%-32.2 \%)$. In the remainder of the population $(n=33779-652=$ $33127)$ coughing was reported by 5875 patients $(17.7 \%$; $95 \%$ CI $17.3 \%-18.1 \%)$. 
Table 1 Comparison of 1458 patients with cough and 4182 controls matched by general practice

\begin{tabular}{lccc}
\hline & $\begin{array}{l}\text { Cases } \\
(n=1458)\end{array}$ & $\begin{array}{l}\text { Controls } \\
(n=4182)\end{array}$ & $\begin{array}{l}\text { OR }(95 \% \text { CI }) \text { or } \\
P \text {-value }\end{array}$ \\
\hline $\begin{array}{l}\text { Mean age (SD) } \\
\text { Females (\%) }\end{array}$ & $50.2(18.2)$ & $46.8(18.0)$ & $<0.001$ \\
Mean no. prescriptions & $903(62)$ & $2572(62)$ & $1.0(0.9-1.2)$ \\
Mean no. consultations & 15.1 & 9.9 & $<0.001$ \\
ACE inhibitors $(\%)^{\mathrm{a}}$ & 15.2 & 11.0 & $<0.001$ \\
Captopril $(\%)^{\mathrm{a}}$ & $48(3.3 \%)$ & $69(1.6 \%)$ & $2.1(1.5-3.1)$ \\
Enalapril $(\%)^{\mathrm{a}}$ & $13(0.9 \%)$ & $29(0.7 \%)$ & $1.3(0.7-2.5)$ \\
Lisinopril $(\%)^{\mathrm{a}}$ & $30(2.1 \%)$ & $36(0.9 \%)$ & $2.6(1.6-4.2)$ \\
Perindopril $(\%)^{\mathrm{a}}$ & $3(0.2 \%)$ & $4(0.1 \%)$ & $2.0(0.5-9.3)$ \\
NSAID $(\%)^{\mathrm{a}}$ & $2(0.1 \%)$ & 0 & - \\
$\beta$-blockers $(\%)^{\mathrm{a}}$ & $410(28 \%)$ & $917(22 \%)$ & $1.4(1.2-1.6)$ \\
Thiazides $(\%)^{\mathrm{a}}$ & $110(7.5 \%)$ & $246(5.9 \%)$ & $1.4(1.1-1.7)$ \\
Ca-antagonists $(\%)^{\mathrm{a}}$ & $15(1.0 \%)$ & $23(0.5 \%)$ & $2.0(1.1-3.9)$ \\
Smoking $(\%)$ & $87(6.0 \%)$ & $124(3.0 \%)$ & $2.2(1.6-2.9)$ \\
\hline
\end{tabular}

${ }^{a}$ Number $(\%)$ of patients exposed on the index date
In total, 6056 patients had coughing as the diagnosis or for whom coughing was recorded in free text. $4499(74.3 \%)$ of these observations were excluded from the analysis for the following reasons: age younger than 20 years $(35 \%)$, being a non-incident case $(36.9 \%)$, suffering from one or more of the aforementioned conditions predisposing to cough $(37.3 \%)$. In principle four controls per case were selected for the remaining 1557 observations. For 99 case patients no control could be found and they were excluded from further analysis. Hence, 1458 case patients comprised the study population. Of the 5832 identified control subjects, 1650 $(28.3 \%)$ were excluded because - age younger than $20 \mathrm{y}(17.9 \%)$, no consultation after the index date of the matching case $(1.8 \%)$, and registration of one or more of the aforementioned conditions predisposing to coughing $(9.5 \%)$. Consequently, the final series consisted of 4182 control subjects, comprising 2-3 matched controls for every case.

A comparison of cases and controls is tabulated in Table 1. Several differences emerged which could explain differences in exposure frequency between cases and control subjects, irrespective of a real association between ACE inhibitors and cough. The cases were somewhat older than the controls, although the gender distribution was similar in the two groups. Cases received significantly more prescriptions and had more consultations per person during the study period than the control group. Patients with cough were more likely to be taking NSAID, $\beta$-adrenoceptor blockers, thiazides and calcium-antagonists. The percentage of smokers was not significantly different between cases and controls, but the figures were lower than expected. Factors were included in the model to study their effect upon the crude estimate.

Of the cases $48(3.3 \%)$ were identified as users of ACE inhibitors, against $69(1.6 \%)$ of the controls. The results of the conditional logistic regression analyses without (crude) and with (adjusted) simultaneous control for confounding variables are presented in Table 2. Cases were 2.1-times more likely than controls to have
Table 2 Crude and adjusted odds ratios for exposure to ACE inhibitors. Exposure odds ratios with a value of unity outside the 95\%-confidence interval are printed in bold type

\begin{tabular}{lll}
\hline & Crude OR & Adjusted OR \\
\hline All ACE inhibitors & $\mathbf{2 . 1}(\mathbf{1 . 5}-\mathbf{3 . 1})$ & $1.4(0.9-2.1)$ \\
Captopril & $1.3(0.7-2.5)$ & $0.9(0.4-1.7)$ \\
Enalapril & $\mathbf{2 . 6}(\mathbf{1 . 6}-\mathbf{4 . 2})$ & $\mathbf{1 . 7}(\mathbf{1 . 0 3 - 2 . 8})$ \\
Lisinopril & $2.0(0.5-9.3)$ & $1.7(0.4-7.9)$ \\
Perindopril & $-\mathbf{2 . 1}(\mathbf{1 . 3}-\mathbf{3 . 5})$ & $1.4(0.8-2.5)$ \\
Females & $\mathbf{2 . 8 ( 1 . 2 - 6 . 6 )}$ & $2.5(1.0-6.3)$ \\
Males & $4.8(\mathbf{1 . 7}-\mathbf{1 3 . 3})$ & $\mathbf{3 . 0}(\mathbf{1 . 0 5}-\mathbf{8 . 3})$ \\
Exposure $<2$ months & $\mathbf{2 . 0}(\mathbf{1 . 1}-\mathbf{3 . 8})$ & $1.2(0.6-2.3)$ \\
Exposure 2-6 months & $1.6(0.9-2.7)$ & $1.0(0.6-1.8)$ \\
Exposure > 6 months & &
\end{tabular}

been exposed to ACE inhibitors (95\% CI 1.5-3.1), but after adjustment the odds ratio declined to $1.4(95 \%$ CI $0.9-2.1$ ). When results were analysed according to the specific ACE inhibitor used, a crude odds ratio of 1.3 (95\% CI 0.7-2.5) was found for captopril, which declined to $0.9(95 \% \mathrm{CI} 0.4-1.7)$ after adjustment. For enalapril the crude odds ratio was $2.6(95 \%$ CI 1.6-4.2) and $1.7(95 \% \mathrm{CI} 1.03-2.8)$ after adjustment. Patients with cough were 2.0 -times ( $95 \%$ CI $0.5-9.3)$ more likely than control patients to have been exposed to lisinopril, and after adjustment the odds ratio was 1.7 (95\% CI 0.4-7.9). Because there were no control patients exposed to perindopril, an odds ratio could not be calculated for this ACE inhibitor.

Subanalyses according to sex yielded the following crude odds ratios: 2.1 (95\% CI 1.3-3.5) and $2.8(95 \%$ CI 1.2-6.6) for females and males, respectively. After adjustment neither value was significant. A doseresponse relationship was not observed for captopril, or for enalapril. For captopril, the crude odds ratio increased from $0.8(95 \% \mathrm{CI} 0.2-3.7)$ in those treated with doses of $25 \mathrm{mg}$ or less per day, and $1.2(95 \% \mathrm{CI}$ $0.4-3.2)$ in those treated with more than $25 \mathrm{mg}$ but less than $75 \mathrm{mg}$ daily, to $2.1(95 \% \mathrm{CI} \mathrm{0.8-5.6)}$ for those patients treated with doses from $75-100 \mathrm{mg}$ per day 
$(P=0.1)$. For enalapril, the crude odds ratio was $2.0(95 \%$ CI $0.7-5.8)$ in those treated with the lowest doses of $5 \mathrm{mg}$ or less per day, 2.9 (95\% CI 1.1-7.8) in patients on $10-20 \mathrm{mg}$ per day, and 2.7 for patients using the highest doses from $20-40 \mathrm{mg}$ per day; $(P=0.9)$.

When overall results were stratified according to the duration of treatment, the odds ratios were $1.6(95 \%$ CI 0.9-2.7) for long term users (> 6 months), 2.0 ( $95 \%$ CI 1.1-3.8) for patients who had been on ACE inhibitor treatment for 2-6 months and $4.8(95 \%$ CI $1.7-13.3)$ for those just starting treatment $(<2$ months). After adjustment, the figures declined and the only significant value was that for starters.

\section{Discussion}

It is very difficult to compare studies of the frequency of ACE inhibitor-induced cough in the literature. This is exemplified by the fact that is has been variously reported in $0.5-39 \%$ of recipients [3]. There are several reasons for this difficulty. First, the risk estimate is given as a straightforward frequency in some studies but as a point prevalence, period prevalence, incidence rate or cumulative incidence in others. Also, the length of the study period has varied. Second, while some studies have relied on spontaneous reporting, in others patients were specially asked about coughing. Third, several studies have comprised case series or small uncontrolled studies with retrospective review of medical records, whereas the two largest studies so far were uncontrolled. Although there have also been some controlled studies, they have been small and not always representative of the use of ACE inhibitors under everyday circumstances. Fourth, the setting has differed from studies of patients hospitalised in tertiary clinics to data gathered in an outpatient setting. Fifth, none of the studies have controlled for the number of consultations per patient. For these reasons, we performed this casecontrol study in a large population under everyday circumstances.

Our crude results suggest that the risk of coughing is increased twofold among patients exposed to ACE inhibitors. But after controlling for confounding bias, the estimate did not remain statistically significantly away from the hull. Crude odd ratios were most influenced by the adjustments for number of consultations, age and use of NSAID and calcium-antagonists. The fact that the cases were older and had more consultations per person, and might thus more easily have been recognised or diagnosed as coughers, explains part of their increased exposure to ACE inhibitors. Although it seems very unlikely that the crude increased risk can be explained solely by these factors, it shows why the number of consultations should be included in all studies of subjective adverse effects based on GP data. The association appeared to be stronger with the longer-acting ACE inhibitors enalapril and lisinopril, but only with enalapril was the adjusted odds ratio still significant. Initially, it was postulated that coughing was probably related to the sulfhydryl group of captopril, but later studies suggested that cough occurred more frequently with enalapril, and postulated that it was related to its longer duration of action [3]. In our current study, we did not find evidence of a relation to gender, as has previously been suggested [4-22]. The risk of coughing was not increased in relation to the dose of captopril and enalapril. This finding is consistent with other reports [3, 22], although some scattered case reports have suggested that a reduction in dosage may sometimes decrease or even abolish cough. Although there were only sufficient data to permit a crude stratification by the duration of treatment, our results suggest a clear duration-effect relationship. According to the literature, cough induced by ACE inhibitors can occur from 24 hours to several months after initiation of therapy [33], and according to some papers no duration-effect relationship can be demonstrated $[5,6,12,34]$. Analysis of captopril and enalaprilinduced cough revealed that the average time to onset was 9 and 4 weeks, respectively $[5,33]$. Even with a variable latency period, however, it seems plausible that the chance that patients will develop coughing will consequently decline if they did not develop it during the first months of treatment. Discontinuation of ACEinhibitors in patients with persistent coughing will consequently lead to a depletion of susceptible persons. Hence, diminution of the association with increasing duration of treatment seems a logical result. Duration of treatment might explain in part the large variation in risk estimates in the literature about the magnitude of this association.

One of the advantages of a study using automated GP data is that information on disease and exposure are gathered by GPs who are not aware of the research hypothesis at the time of registration. Hence, no selection of cases can be made by the GP. Also, selection of control subjects was performed at random and was independent of drug use. It is possible, however, that selection bias may account for the slightly higher risk of coughing after enalapril than captopril in our study, if doctors were liable to try a newer ACE-inhibitor in patients who developed coughing to older ACEinhibitors. It must be emphasised that not all patients will consult their GP because of coughing, and so there might be underestimation of the risk if some of the controls had cough due to an ACE-inhibitor without consulting their GP. This does not seem to be very likely, however, for two reasons. First, to control for this potential bias, the control group consisted of patients who had had at least one consultation with their GP in the same period and second, as the dry tickling cough to ACE-inhibitors is burdensome and very persistent, controls would have presented themselves to the GP sooner or later and would thus have been registered as cases. 
A major concern in a case-control study is possible information bias. Recall bias was not very likely to occur as the case-control study made use of data which had already been gathered before the onset of the disease. Another type of information bias might occur, however, if GPs were more likely to diagnose coughing in users of ACE inhibitors than in other patients. During registration and classification of symptoms and diseases, cough is a well-known adverse effect of ACE inhibitor treatment, so it is possible that some selection in registration might have occurred. Information bias could also be the result of patients who presented themselves to their GPs with coughing after reading the data sheet. A third point is the evaluation of misclassification of disease and exposure. As we were unable to confirm the relationship between coughing and use of captopril - the first ACE-inhibitor and probably the most wellknown cause of coughing - such information bias is unlikely. Cough is a very general and poorly defined symptom. In fact, we were only interested in a particular type of cough; the non-productive, tickling cough. For this type of subjective diagnosis, the International Classification of Primary Care is insufficiently subtle to describe the patient's condition. Cases of ACE inhibitorinduced cough may not have been recognized as such, and so could have been misclassified as acute respiratory disease, bronchitis, asthma or deteriorating cardiac failure. As patients with such diagnoses were also excluded from the control group, this does not necessarily jeopardise valid estimation of the odds ratio. The criteria for defining exposure also require consideration. Subjects were only considered as exposed if the index date fell between the start and stop date of the total estimated exposure experience. Subjects who received a prescription for an ACE inhibitor outside the time frame between this index date and the beginning of the data collection period may have been misclassified as non-exposed. As the average period between coughing and the start of the study period was almost 10 months, however, such misclassification is unlikely. Subjects may also have been misclassified as non-exposed, if they received a prescription from an ACE inhibitor from a medical specialist, as such prescriptions were not registered in the GP data base. Although GPs usually take over the treatment of such patients after one or several consultations, such misclassification might have led to a slight bias of the risk estimation towards the null hypothesis. Unfortunately, smoking habits are poorly registered in GP databases but in an earlier study we did not find a relationship between smoking and exposure to ACE-inhibitors [22].

In conclusion, we found that the risk for coughing due to ACE-inhibitors was highest in the first two months of exposure that it declined with duration of exposure. This could be explained by the assumption that susceptible persons were changed to other therapy. It is possible that such depletion of susceptibles may partly explain the lower risk in users of captopril than in patients on enalapril.

\section{References}

1. Havelka J, Vetter H, Studer A, Greminger $P$, Luscher $T$, Wollnik S, Siegenthaler W, Vetter W (1982) Acute and chronic effects of Angiotensin-Converting Enzyme Inhibitor Captopril in Severe Hypertension. Am $\mathbf{J}$ Cardiol 49: 1467-1474

2. Sesoko S, Kaneko Y (1985) Cough associated with the use of captopril. Arch Intern Med 145:1524

3. Israili ZH, Hall WD (1992) Cough and angioneurotic edema associated with angiotensin-converting enzyme inhibitor therapy. A review of the literature and pathophysiology. Ann Intern Med 117:234-242

4. Inman WHW (1986) Enalapril-induced cough. Lancet 2:1218

5. Coulter DM, Edwards IR (1987) Cough associated with captopril and enalapril. BMJ 294:1521-1523

6. Yeo WW, Ramsey LE (1990) Persistent dry cough with enalapril: incidence depends on method used. J Hum Hypertens $4: 517-520$

7. Stoller JK, Elghazawi A, Mehta AC, Vidt DG (1988) Captoprilinduced cough. Chest 93:659-661

8. Webb D, Benjamin N, Collier J, Robinson B (1986) Enalaprilinduced cough. Lancet 2:1094

9. Kaufman J, Casanova JE, Riendl P, Schlueter DP (1989) Bronchial hyperreactivity and cough due to angiotensin-converting enzyme inhibitors. Chest 95:544-548

10. Hume AL, Murphy JL, Lauerman SE (1989) Angiotensin-converting enzyme inhibitor-induced cough. Pharmacotherapy 9 : $88-90$

11. Morice AH, Brown MJ, Higenbottam T (1989) Cough Associated with Angiotensin Converting Enzyme Inhibition. J Cardiovase Pharmacol 13 [Suppl 3]:S59-S62

12. McEwan JR, Choudry N, Street R, Fuller RW (1989) Change in cough reflex after treatment with enalapril and ramipril. BMJ 299:13-16

13. Yeo WW, Foster G, Ramsay LE (1991) Prevalence of persistent cough during long-term enalapril treatment: controlled study versus nifedipine. Q J Med 80: 763-770

14. Poole MD, Postma DS (1991) Characterization of cough associated with angiotensin converting enzyme inhibitors. Otolaryngol Head Neck Surg 105:714-716

15. Yeo WW, Maclean D, Richardson PJ, Ramsey LE (1991) Cough and enalapril: assessment by spontaneous reporting and visual analogue scale under double-blind conditions. $\mathrm{Br} \mathrm{J}$ Clin Pharmacol 31:356-359

16. Os I, Bratland B, Dahlof B, Gisholt K, Syvertsen JO, Tretli S (1992) Female sex as an important determinant of lisinoprilinduced cough. Lancet 339:372

17. Strocchi E, Valtancoli, G, Ambrosioni E (1989) The incidence of cough during treatment with angiotensin converting enzyme inhibitors. J Hypertens 7 [Suppl 6]:S308-S309

18. Moore N, Noblet C, Joannides R, Ollagnier M, Imbs JL, Lagier G (1993) Cough and ACE inhibitors. Lancet 341:61

19. Lefebvre J, Poirier L, Lacourciere Y (1992) Prospective trial on captropril-related cough. Ann Pharmacother 26:161-164

20. Carre A, Vasmant D, Elmalem J, Thiery P (1991) Tolerability of ramipril in a multicenter study of mild-to-moderate hypertension in general practice. J Cardiovase Pharmacol 18 [supp] 2]:S141-S143

21. Strocchi E, Valtancoli G, Ricci C, Malini PL, Bassein L, Ambrosioni E (1992) Postmarketing studies of subjective side effects; a case for strict methodological criteria and careful analysis of data. Pharmacol Res 25 [Suppl 1]:79-80

22. Visser LE, Stricker BHCh, Van der Velden J, Paes AHP, Bakker A (1995) ACE-inhibitor associated cough: a population-based case-control study. J Clin Epidemiol 48:851-857

23. Inman WHW, Rawson NSB, Wilton LV, Pearce GL, Speirs CJ (1988) Postmarketing Surveillance of enalapril. 1: Results of Prescription Event-Monitoring. BMJ 297:286-289

24. Cooper WD, Sheldon D, Brown D, Kimber GR, Isitt VL, Currie WJ (1987) Post-marketing surveillance of enalapril: 
experience in 11,710 hypertensive patients in general practice. J R Coll Gen Pract 37:346-349

25. Van der Lei J, Duisterhout JS, Westerhof HP, Van der Does E, Cromme PVM, Boon WM, Van Bemmel JH (1993) The introduction of computer-based patient records in the Netherlands. Ann Int Med 119:1036-1041

26. Lamberts H, Woods M (1987) International classification of primary care. Oxford University Press, Oxford

27. Anonymous. (1987) Anatomical therapeutic chemical (ATC) index: Including defined daily doses (DDD) for plain substances. World Health Organization, Oslo

28. Puolijoki HJ, Nieminen MM, Siitonen LO, Lahdensuo AH, Reinikainen PM (1989) Is a simultaneous beta-blocker therapy a risk factor for enalapril-induced cough? Respiration 55: $127-128$

29. Kamei J, Kasuya Y (1992) The effect of hydrochlorothiazide on the enhanced coughing associated with treatment with enalapril. Eur J Pharmacol 213:137-139
30. Fogari R, Zoppi A, Tettamanti F, Malamani GD, Tinelli C, Salvetti A (1992) Effects of nifedipine and indomethacin on cough induced by angtiotensin converting enzyme inhibitors: a double-blind radomized, cross-over study, J Cardiovase Pharmacol 19:670-673

31. Kleinbaum DG, Kupper LL, Muller KE (1988) Applied regression analysis and other multivariate methods. PWS-Kent Publishing Company, Boston

32. Egret. $₫$ Statistics and Epidemiology Research Corporation, Seattle, WA.

33. Just PM (1989) The positive association of cough with angiotensin-converting enzyme inhibitors. Pharmacotherapy 9 : $82-87$

34. Sebastian JL, Mc Kinney WP, Kaufman J, Young MJ (1991) Angiotensin converting enzyme inhibitors and cough. Chest $99: 36-39$ 